\title{
Holomorphic Extension from Weakly Pseudoconcave CR Manifolds
}

\author{
Andrea Altomani (*) - C. Denson Hill (**) - Mauro Nacinovich (***) \\ Egmont Porten $(* *)$
}

ABSTRACT - Let M be a smooth locally embeddable CR manifold, having some CR dimension $m$ and some CR codimension $d$. We find an improved local geometric condition on $M$ which guarantees, at a point $p$ on $\mathrm{M}$, that germs of CR distributions are smooth functions, and have extensions to germs of holomorphic functions on a full ambient neighborhood of $\mathrm{p}$. Our condition is a form of weak pseudoconcavity, closely related to essential pseudoconcavity as introduced in [HN1]. Applications are made to CR meromorphic functions and mappings. Explicit examples are given which satisfy our new condition, but which are not pseudoconcave in the strong sense. These results demonstrate that for codimension $d>1$ there are additional phenomena, which are invisible when $d=1$.

\section{Introduction.}

The goal of the present article is to give improved geometric conditions on a generic $\mathrm{CR}$ manifold $M \subset \mathrm{C}^{n}$ which guarantee that all local $\mathrm{CR}$ functions extend holomorphically to a full neighborhood of a given point. This is well known to be true for strictly pseudoconcave CR manifolds,

(*) Indirizzo dell'A.: Research Unity in Mathematics, University of Luxembourg, 6, rue Coudenhove-Kalergi, L-1359 Luxembourg.

E-mail address: andrea.altomani@uni.lu

(**) Indirizzo dell'A.: Department of Mathematics, Stony Brook University, Stony Brook NY 11794 (USA).

E-mail address: dhilll@math.sunysb.edu

(***) Indirizzo dell'A.: Dipartimento di Matematica, II Università di Roma "Tor Vergata", Via della Ricerca Scientifica, 00133 Roma (Italy).

E-mail address: nacinovi@mat.uniroma2.it

$(* *$ ) Indirizzo dell'A.: Department of Mathematics, Mid Sweden University, 85170 Sundsvall (Sweden)

E-mail address: Egmont.Porten@miun.se 
i.e. in the case where the Levi form has one negative eigenvalue in each characteristic conormal direction. For hypersurfaces this is a classical result of $\mathrm{H}$. Kneser and $\mathrm{H}$. Lewy; for $M$ of higher codimension it was proved independently by a number of authors (see [BP], [NV] for CR distributions, and [HN4] for two different proofs, one very short). Despite numerous efforts, the general problem to characterize those weakly pseudoconcave manifolds for which one has extension to full neighborhoods is still far from being completely understood, even for real analytic hypersurfaces.

Subtle sufficient conditions (sector and ray property) are known for weakly pseudoconcave hypersurfaces of finite type (see [BT2], [FR] for results and references). In higher codimension there are several options to approach the weakly pseudoconcave case. Manifolds which are Levi flat at the reference point to a certain order, and have all relevant concavity in the generalized Levi form determined by the next-order terms are studied in [Bo]. Here we aim at the opposite case where effects of different orders (counted with respect to bracket length) are combined. Actually our main motivation stems from homogeneous CR manifolds which biholomorphically look the same near every point. These higher codimensional homogeneous CR manifolds are abundant, occurring naturally in mathematics, and they have a strong tendency to be weakly pseudoconcave (see [MN1], [MN2], [MN3], [MN4], [AMN]). The main result of the present article reveals that there are additional phenomena which are invisible in codimension one, and indicates that finite type together with a suitable notion of weak pseudoconcavity should imply extension to a full neighborhood. To avoid confusion, we stress that the problem under consideration is different in nature from the problem of holomorphic wedge extension, for which a definitive answer is known ([T1], [Tu1], [J], $[\mathrm{M}]$, see also [MP2]). In fact, this definitive answer was obtained without having explicit control on the directions of extension, which is crucial for the problem at hand.

Let $M \subset \mathbb{C}^{n}$ be a smooth CR manifold. We denote by $J$ the complex structure tensor on $T \mathrm{C}^{n}$, by $H M=T M \cap J T M$ the holomorphic tangent bundle of $M$, which is the real subbundle of $T M$ invariant under $J$, and by $H^{0} M \subset T^{*} M$ the characteristic bundle, defined fiberwise as the annihilator of $H M$. We define the vector valued Levi form

$$
\mathcal{L}_{M, p}=\mathcal{L}_{p}: H_{p} M \times H_{p} M \rightarrow \mathrm{C} \otimes\left(T_{p} M / H_{p} M\right)
$$

by

$$
\mathcal{L}_{M, p}(X, Y)=-[\tilde{X}, J \tilde{Y}](p)+i[\tilde{X}, \tilde{Y}](p) \bmod \mathbb{C} \otimes H_{p} M,
$$


where $\tilde{X}, \tilde{Y} \in \Gamma(M, H M)$ are smooth extensions of $X, Y$, respectively. The usual Levi form $\mathcal{L}_{M, \xi}=\mathcal{L}_{\xi}$ is parameterized by the characteristic codirections $\xi \in H_{p}^{0} M$. It is defined by $\mathcal{L}_{M, \xi}(X, Y)=\xi\left(\mathcal{L}_{p}(X, Y)\right)$, for $\xi \in H_{p}^{0} M$, $X, Y \in H_{p} M$ (where we read $\xi$ as a form on $\mathbb{C} \otimes\left(T_{p} M / H_{p}^{0} M\right)$ in the canonical way). Most often we shall work with the corresponding real hermitian forms $\mathcal{L}_{p}(X)=\mathcal{L}_{p}(X, X) \in T_{p} M / H_{p} M$ and $\mathcal{L}_{\xi}(X)=\mathcal{L}_{\xi}(X, X)$. The reader should take note of the subtle difference in notation between $\mathcal{L}_{p}(X)$ and $\mathcal{L}_{\xi}(X)$; the former is vector valued, and the latter is scalar valued.

A CR manifold $M$ is strictly (weakly) pseudoconcave at a point $p \in M$ if for every $\xi \in H_{p}^{0} M, \xi \neq 0, \mathcal{L}_{\xi}$ has a negative (nonpositive) eigenvalue. Replacing $\xi$ by $-\xi$, we see that $\mathcal{L}_{\xi}$ has actually eigenvalues of both signs (in the strictly pseudoconcave case). Following [HN1], we call $M$ trace pseudoconcave at $p \in M$ if for every $\xi \in H_{p}^{0} M, \mathcal{L}_{\xi}$ is either zero or has eigenvalues of both signs. Trace pseudoconcavity isolates one of the properties of essential pseudoconcavity introduced in [HN1]. We refer to that article for background information.

Let $\mathcal{G}_{1}$ be the sheaf of germs of smooth (real) $\mathrm{CR}$ vector fields on $\mathrm{M}$ (i.e. sections of $H M$ ). For every positive integer $k$ we define inductively $\mathcal{G}_{k+1}$ as the sheaf generated by $\mathcal{G}_{k}$ and $\left[\mathcal{G}_{1}, \mathcal{G}_{k}\right]$. Let $G_{k, p} \subset T_{p} M$ be the vector space generated by pointwise evaluations of germs in $\mathcal{G}_{k}$ at a point $p \in M$. We say that $M$ is of $k$ ind $k$ at $p$ if $G_{k, p}=T_{p} M$ but $G_{j, p} \mp T_{p} M$ for $j<k$. We say that $M$ satisfies the constant rank condition if the spaces $G_{k, p}$ have dimension independent of $p$, i.e. if they form vector bundles $G_{k}=\bigcup G_{k, p}$.

Now we can formulate our main result.

THEOREM 1.1. Let $M$ be a smooth generic $C R$ manifold in $\mathbb{C}^{n}$ and $p_{0} \in M$. Assume that in a neighborhood of $p_{0}, M$ is trace pseudoconcave, satisfies the constant rank condition and is of kind less or equal to 3. Then for every open neighborhood $U$ of $p_{0}$ in $M$, there is an open neighborhood $V$ of $p_{0}$ in $\mathbb{C}^{n}$ such that every $C R$ distribution on $U$ is smooth on $M \cap V$ and has a unique holomorphic extension to $V$.

We emphasize that Theorem 1.1 reveals a phenomenon which remains invisible in codimension 1. In fact, in the hypersurface case its assumptions imply that $M$ is of kind 2, hence strictly pseudoconcave. We expect the result to extend to arbitrary finite kind. In [HN1], the weak identity principle for CR functions (coincidence on open sets implies coincidence everywhere) was shown for essentially pseudoconcave CR manifolds. For those CR manifolds covered by the assumptions of Theorem 1.1 our result immediately yields the strong identity principle 
(coincidence of Taylor coefficients at some point yields coincidence in a neighborhood).

Theorem 1.1 is proved in Sections 2-4. The rest of the article is devoted to applications, extensions and examples. In Section 5 we observe that CR manifolds as in Theorem 1.1 enjoy the local extension property $\mathrm{E}$ introduced in [HN2]. The results in [HN2], [HN3] yield far reaching global consequences for fields of CR meromorphic functions on such manifolds. Here CR meromorphic functions are functions which are locally representable as fractions of $\mathrm{CR}$ functions. An alternative approach to $\mathrm{CR}$ meromorphic mappings originates from work of Harvey and Lawson [HL]. The idea is to require the graph to look like a CR manifold with appropriate singularities. In general, extension of such mappings is complicated. Based on [MP2], we prove in Section 6 that such CR meromorphic functions extend meromorphically from manifolds with property $\mathrm{E}$ to full ambient neighborhoods and are in particular representable as local quotients. In Section 7 we present several classes of homogeneous CR manifolds to which all the local and global results indicated above apply. These were discovered in a much broader context (see [AMN]). For the reader's comfort, we give a reasonably self-contained presentation.

\section{Preliminaries.}

We will use some standard facts about the bundles $G_{1} \subset G_{2} \subset \ldots$ If $G_{k}=G_{k+1}$ then all $G_{j}, j \geq k$, are equal (the proof is an application of the Jacobi identity). This means in particular that $G_{k}$ is integrable in the sense of Frobenius. Moreover the map associating to smooth sections $X \in \Gamma\left(U, G_{1}\right), Y \in \Gamma\left(U, G_{k}\right)$, the section $[X, Y] \bmod G_{k} \in \Gamma\left(U, G_{k+1} / G_{k}\right)$ is tensorial, i.e. $[X, Y](p) \bmod G_{k, p}$ depends only on $X(p)$ and $Y(p)$.

Let us now have a closer look at $G_{2}$. First we note that independently of concavity $G_{2, p} / H_{p} M$ is spanned as a real vector space by the image $C_{p}=\left\{\mathcal{L}_{p}(X): X \in H_{p} M\right\}$ of the vector valued Levi form. Indeed, $L=\operatorname{span}_{\mathbb{R}} C_{p}$ is contained in $G_{2, p} / H_{p} M$ by definition. On the other hand, polarization shows that $L \otimes \mathrm{C}=\operatorname{span}_{\mathrm{C}}\left\{\mathcal{L}_{p}(X, Y): X, Y \in H_{p} M\right\}$. Since the imaginary part of $\mathcal{L}_{p}(X, Y)$ is essentially $[X, Y]$, we obtain $G_{2, p} / H_{p} M \subset L$. The above is equivalent to the fact that $G_{2, p}$ is spanned by the preimage of $C_{p}$ under the canonical projection $T_{p} M \rightarrow T_{p} M / H_{p} M$.

A simple but crucial observation is that trace pseudoconcavity allows us to replace linear spans by convex hulls. 
Lemma 2.1. Assume that $M$ is trace pseudoconcave at $p \in M$. Then $G_{2, p} / H_{p} M$ is the convex hull of $C_{p}=\left\{\mathcal{L}_{p}(X): X \in H_{p} M\right\}$.

PRoof. If the lemma fails, there is a nonzero linear functional $\xi$ on $G_{2, p} / H_{p} M$ such that $C_{p} \subset\{\xi \geq 0\}$. We may extend $\xi$ to an element of $H_{p}^{0} M$. Since $G_{2, p} / H_{p} M$ is the linear span of $C_{p}$, there is an $X \in C_{p}$ with $\xi(X)>0$. Any $Y \in H_{p} M$ with $\mathcal{L}_{p}(Y)=X$ satisfies $\mathcal{L}_{\xi}(Y)>0$. But this implies that $\mathcal{L}_{\xi}$ has also some negative eigenvalue, in contradiction to $C_{p} \subset\{\xi \geq 0\}$

To keep track of directions of extension, we will use an analogue of the analytic wave front set, denoted by $W F_{u}$. It is defined for CR distributions $u$ via the FBI transform in [S], see also [T2]. For $U$ open in $M$, let $C R(U)$ denote the space of continuous $\mathrm{CR}$ functions defined on $U$. We do not even have to recall the definition of $W F_{u}$, since the following basic properties will suffice for our purposes:

(a) Let $u$ be a CR distribution defined on $U \subset M$. Then $W F_{u}$ is a cone, closed in the pointed characteristic bundle $H^{0} U \backslash o$ (o denoting the zero section).

(b) $W F_{u} \cap H_{p}^{0} M=\emptyset$ holds if and only the CR distribution $u$ extends holomorphically to an ambient neighborhood of $p$.

(c) Let $u \in C R(U)$. If $\mathrm{CR}$ extension from $U$ holds at $(p, X), p \in U$, $X \in T_{p} M \backslash H_{p} M$, then for any $\xi \in W F_{u} \cap H_{p}^{0} M$ we have $\xi(X) \geq 0$.

In (c) we use the following terminology: We say that $C R$ extension from $U$ holds at $(p, X), p \in U, X \in T_{p} M \backslash H_{p} M$, if there is a $\mathcal{C}^{2}$-smooth ( $\left.\operatorname{dim} M+1\right)$ dimensional CR manifold $\tilde{M}$ attached to $U$ along some $U$-neighborhood $U^{\prime}$ of $p$ such that (i) for a representative of $X, J X$ points into $\tilde{M}$ and (ii) every $u \in C R(U)$ has a continuous extensions to $\tilde{M} \cup U^{\prime}$ which is CR on $\tilde{M}$. Neglecting the dependence on $U$, we will sometimes call $(p, X)$ or just $X$, a direction of CR extension. For (a), (b), see [S], whereas (c) is observed in [T2].

Theorem 1.1 is a consequence of the following more precise result which does not require kind 3 .

THEOREM 2.2. $\quad$ Let $M$ be a smooth generic $C R$ manifold in $\mathrm{C}^{n}$. Assume that on an open set $U \subset M, M$ is trace pseudoconcave and that $G_{2}, G_{3}$ are bundles. Then for every continuous $C R$ function $u$ defined on $U$, we have $W F_{u} \subset G_{3}^{\perp}$.

Theorem 2.2 will be proved in the next two sections. It implies Theorem 1.1 in the following way: Kind 3 means that $G_{3}^{\perp}$ is the zero bundle near 
$p_{0}$. By (b) a continuous CR function $u$ extends holomorphically to an ambient neighborhood of every $p$ contained in some neighborhood $U^{\prime}$ of $p_{0}$ in $M$. By a standard gluing argument one obtains extension to an ambient neighborhood $V^{\prime}$ of $U$, which may a priori depend on $u$. Note that holomorphic extension in particular shows that $u \in \mathcal{C}^{\infty}\left(U^{\prime}\right)$. Now a Baire category argument as in [HN2] yields extension to a neighborhood of $V$ whose size only depends on $U$. This proves the theorem for continuous $\mathrm{CR}$ functions.

If $u$ is a CR distribution, we may use a method from [BT1], [T], to represent it near $p_{0}$ as $u=\Delta_{M}^{k} f$, where $f$ is a continuous CR function. Here $k$ is a sufficiently large integer, and $\Delta_{M}$ is a variant of the Laplace operator which is defined in an ambient neighborhood of $p_{0}$ and restricts nicely to $M$. If $\tilde{f}$ is a holomorphic extension of $f$, then the various properties of $\Delta_{M}$ imply that $\Delta_{M}^{k} \tilde{f}$ is the desired extension of $u$. Hence Theorem 1.1 follows from Theorem 2.2.

\section{Proof plan for Theorem 2.2.}

Here we will prove Theorem 2.2 modulo some more technical results on $\mathrm{CR}$ extension which are postponed to the following section. Pick some $u \in C R(U)$.

STEP 1. $-W F_{u} \subset G_{2}^{\perp}$. This will follow from trace pseudoconcavity. It is a consequence of the following lemma which holds without constant rank assumptions.

Lemma 3.1. Assume that $M \subset \mathbb{C}^{n}$ is trace pseudoconcave at $p \in M$. Then for every continuous CR function $u$ defined near $p$ we have $W F_{u} \cap H_{p}^{0} M \subset G_{2, p}^{\perp}$.

Proof. Let $\xi \in W F_{u} \cap H_{p}^{0} M$. We will consider $\xi$ both as a functional acting on $T_{p} M$ and $T_{p} M / H_{p} M$. By [Tu2], every element $X \in C_{p}$ can be approximated by directions of $\mathrm{CR}$ extensions $X_{j} \in T_{p} M / H_{p} M$. From property (c) and continuity we get $\xi(X) \geq 0$. It follows that $\xi$ is nonnegative on the convex hull of $C_{p}$. Since this convex hull is the vector space $G_{2, p} / H_{p} M, \xi$ vanishes on $G_{2, p}$

Notice that the lemma together with property (b) already imply extension to a full neighborhood for strictly pseudoconcave CR manifolds. 
SteP 2. $-W F_{u} \subset G_{3}^{\perp}$. By Step 1 it suffices to show that a covector $\xi_{0}$ that annihilates $G_{2}$ but not $G_{3}$ is not contained in $W F_{u}$. Let $p_{0} \in U$ denote the base point to which $\xi_{0}$ projects. It is our aim to show $\xi_{0} \notin W F_{u}$ by constructing an appropriate CR extension and applying property (c).

Since the image $C_{p_{0}}$ of the vector valued Levi form spans $G_{2, p_{0}} / H_{p_{0}} M$ (see the remarks before Lemma 2.1), we may select vectors $X_{1}, \ldots, X_{k} \in H_{p_{0}} M$ such that the vectors $\tilde{Y}_{j}=\mathcal{L}_{M, p_{0}}\left(X_{j}\right), j=1, \ldots, k$, form a basis of $G_{2, p_{0}} / H_{p_{0}} M$. Extending the $X_{j}$ smoothly to CR vector fields defined near $p_{0}$, we obtain a local basis $\tilde{Y}_{j}(p)=\mathcal{L}_{M, p}\left(X_{j}\right)$ of $G_{2} / H M$. Set $Y_{j}=\left[J X_{j}, X_{j}\right]$, and choose a local basis $Z_{1}, \ldots, Z_{2 m}$ of $H M$. Then the $Z_{i}$ form together with the the $Y_{j}$ a local basis of $G_{2}$.

First we claim that $G_{3}$ is spanned in some neighborhood of $p_{0}$ by the $Z_{i}$, $Y_{j}$, together with the brackets $\left[Z_{i}, Y_{j}\right]$. Indeed, by definition $G_{3, p}$ is spanned by $G_{2, p}$ and vectors of the form $[Z, Y](p)$ where $Z \in \mathcal{G}_{1, p}, Y \in \mathcal{G}_{2, p}$ for $p$ near $p_{0}$. Around $p$ we may write $Z=\sum z_{i} Z_{i}, Y=\sum \tilde{z}_{i} Z_{i}+\sum y_{j} Y_{j}$, with smooth coefficients $z_{i}, \tilde{z}_{i}, y_{j}$. This yields

$$
[Z, Y]=\sum z_{i} y_{j}\left[Z_{i}, Y_{j}\right]+R,
$$

where $R$ is a germ in $\mathcal{G}_{2, p}$. This proves the claim.

In the sequel, we will only need the following consequence: Since $\xi_{0}$ does not annihilate $G_{3, p_{0}}$, there are $i_{0}, j_{0}$ such that $\xi_{0}\left(\left[Z_{i_{0}}, Y_{j_{0}}\right]\left(p_{0}\right)\right) \neq 0$. For notational convenience we will write from now on $Y=Y_{j_{0}}, Z=Z_{i_{0}}$.

The following proposition, which will be proved in Section 4, yields CR extension at $\left(p_{0}, Y\left(p_{0}\right)\right)$ realized by a $\mathrm{CR}$ manifold to which $Y$ is complex tangent in a neighborhood of $p_{0}$ in $M$.

Proposition 3.2. Let $M \subset \mathbb{C}^{n}$ be a smooth generic $C R$ manifold of $C R$ dimension $m$ and codimension $d$. Let $p_{0} \in M$ and let $U$ be an open neighborhood of $p_{0}$ in $M$. Let $X$ be a smooth $C R$ vector field on $U$ with $\mathcal{L}_{M, p_{0}}(X) \neq 0$. Then there is a local $\mathcal{C}^{4}$-smooth generic $C R$ manifold $\tilde{M}$ of dimension $\operatorname{dim} M+1$ with the following properties:

(a) $M \cap \tilde{M}$ is a neighborhood of $p_{0}$ in $U$ and $\tilde{M} \backslash M$ has two connected components $\tilde{M}^{ \pm}$.

(b) The distribution on $M \cap \tilde{M}$ spanned by $H M$ and $[J X, X]$ coincides with $\left.(H \tilde{M} \cap T M)\right|_{M \cap \tilde{M}}$.

(c) Denoting by $\tilde{M}^{+}$the side into which $J[J X, X]\left(p_{0}\right)$ points, we have $C R$ extension from $U$ to $\tilde{M}^{+}$.

Since $\xi_{0}$ annihilates $H_{p_{0}} \tilde{M} \cap T_{p_{0}} M$, there is a unique extension $\tilde{\xi}_{0} \in H_{p_{0}}^{0} \tilde{M}$. 
We claim that $\mathcal{L}_{\tilde{M}, \tilde{\xi}_{0}}$ has eigenvalues of both signs. Indeed, both $Z$ and $Y$ may be extended to $\mathcal{C}^{3}$-smooth CR vector fields on $\tilde{M}$ which we denote by the same symbols. For $Y$ we use here that $Y$ is complex tangent to $\tilde{M}$ along $M$ by Proposition 3.2. The fact that $\xi_{0}$ annihilates the image of the Levi form of $M$ implies $\mathcal{L}_{\tilde{M}, \tilde{\xi}_{0}}\left(Z\left(p_{0}\right)\right)=\mathcal{L}_{M, \xi_{0}}\left(Z\left(p_{0}\right)\right)=0$. Since $\tilde{\xi}_{0}\left([Z, Y]\left(p_{0}\right)\right) \neq 0$ is essentially the imaginary part of the sesquilinear Levi form, $Z$ does not lie in the kernel of $\mathcal{L}_{\tilde{M}, \tilde{\xi}_{0}}$. Hence $\mathcal{L}_{\tilde{M}, \tilde{\xi}_{0}}(\cdot)$ takes both positive and negative values in every neighborhood of $Z\left(p_{0}\right)$ in $H_{p_{0}} \tilde{M}$. The claim follows.

Let $X^{+}, X^{-}$be positive and negative eigenvectors of $\mathcal{L}_{\tilde{M}, \tilde{\xi}_{0}}$, respectively. Since all vectors in $H_{p_{0}} M$ are null vectors of $\mathcal{L}_{\tilde{M}, \tilde{\xi}_{0}}$, we may assume, after replacing $X^{ \pm}$by an appropriate complex multiple if necessary, that $X^{ \pm}$ both point into $\tilde{M}^{+}$. But now the following proposition, whose proof is postponed to Section 4, yields for $M$ two directions of CR extension $Y^{ \pm} \in T_{p_{0}} M / H_{p_{0}} M$ with $\xi_{0}\left(Y^{+}\right)>0$ and $\xi_{0}\left(Y^{-}\right)<0$. This implies $\xi_{0} \neq W F_{u}$ by property (c). Hence the proof of Theorem 2.2, and thus also of Theorem 1.1 , will be complete as soon as we have shown the following.

Proposition 3.3. Let $M, \tilde{M}, \tilde{M}^{ \pm}$and $p_{0} \in M$ be as above. Assume that there is $\tilde{\xi} \in H_{p_{0}}^{0} \tilde{M}$ and $X \in H_{p_{0}} \tilde{M} \backslash T_{p_{0}} M$ pointing into $\tilde{M}^{+}$such that $\mathcal{L}_{\tilde{M}, \tilde{\xi}}(X)>0$. Then there is a direction $Z \in T_{p_{0}} M / H_{p_{0}} M$ of $C R$ extension from $U$ satisfying $\tilde{\xi}(Z)>0$.

\section{CR extension.}

In this section we will prove Propositions 3.2 and 3.3. First we recall some basic material on Bishop discs introduced in the seminal paper [B]. Consider a generic $\mathrm{CR}$ manifold $M \subset \mathbb{C}^{n}$ of $\mathrm{CR}$ dimension $m$ and codimension $d$. A $\mathcal{C}^{*}$-smooth analytic disc is a mapping $A(\zeta)=(Z(\zeta), W(\zeta)) \in \mathcal{C}^{*}\left(\bar{\Delta}, \mathbb{C}^{n}\right) \cap \mathcal{O}\left(\Delta, \mathbb{C}^{n}\right)$ where $\Delta=\{\zeta \in \mathbb{C}:|\zeta|<1\}$. We say that $A$ is attached to $M$ if $A\left(S^{1}\right) \subset M$.

For $|\mu| \leq 1$ let $\mathcal{T}_{\mu}$ denote the Hilbert transform of a function $U: S^{1} \rightarrow \mathbb{R}$ to its harmonic conjugate $\mathcal{T}_{\mu} U$, normalized by the condition that the harmonic extension of $\mathcal{T}_{\mu} U$ vanishes at $\zeta=\mu$. It is known that $\mathcal{T}_{\mu}$ is a continuous linear operator on the Hölder spaces $\mathcal{C}^{k, \alpha}\left(S^{1}, \mathbb{R}\right)$ if $k \in \mathbb{N}_{0}$, $0<\alpha<1$. We shall use the same notation for the Hilbert transform applied componentwise to vector valued functions of $\mathcal{C}^{k, \alpha}\left(S^{1}, \mathbb{R}^{d}\right)$.

We work in coordinates

$$
z_{1}=x_{1}+i y_{1}, \ldots, z_{m}=x_{m}+i y_{m}, w_{1}=u_{1}+i v_{1}, \ldots, w_{d}=u_{d}+i v_{d}
$$


centered at the origin in which $M$ is locally given as a graph $v=h(z, u)$ with $h(0)=0, d h(0)=0$. The Bishop equation is the nonlinear functional equation

$$
U=-\mathcal{T}_{\mu}(h(Z, U))+u .
$$

Here $U=U(\zeta)$ is the unknown function mapping the unit circle $S^{1}=\{|\zeta|=1\} \subset \mathbb{C}$ to $\mathbb{R}^{d}$, whereas $Z=Z(\zeta)$ is the boundary value of a given holomorphic function from $\Delta$ to $\mathbb{C}^{m}$ and $u \in \mathbb{R}^{d}$ is a prescribed vector. It is known that the Bishop equation can be solved in $\mathcal{C}^{k, \alpha}\left(S^{1}, \mathbb{R}^{d}\right)$ provided the data $Z(\zeta)$ are $\mathcal{C}^{k, \alpha}$-small and $|u|$ is small (see [MP4] for detailed information). The solution $U$ corresponds to a unique analytic disc $A: \bar{\Delta} \rightarrow \mathbb{C}^{n}$ whose restriction to $S^{1}$ is $(Z(\zeta), U+i h(Z(\zeta), U(\zeta)))$. More precisely, $A$ is holomorphic on $\Delta$ and $\mathcal{C}^{k, \alpha}$-smooth up to $S^{1}$. By construction, $A$ is attached to $M$.

Proof of Proposition 3.2. - The construction of $\tilde{M}^{+}$will be a refinement of [HT, proof of Theorem 9.1]. We may assume $p_{0}=0$ and choose coordinates $(z, w)$ as in (1). After appropriate rotations and dilations in $z$ and $w$, we may furthermore assume $\mathcal{L}_{M, 0}\left(\frac{\partial}{\partial x_{1}}\right)=\frac{\partial}{\partial u_{1}} \bmod H_{0} M$. We will construct $\tilde{M}^{+}$as the union of real curves $\gamma_{p}(s), s \in\left[0, \varepsilon_{1}\right)$, having their initial points at $\gamma_{p}(0)=p \in M$ and such that the segments $\gamma_{p}\left(\left(0, \varepsilon_{1}\right)\right)$ foliate $\tilde{M}^{+}$.

Let us start by the construction of $\gamma_{0}$. Following [HT], we first simplify the defining equations by removing some of the pure terms. After a change of the $w_{1}$-variable

$w_{1} \mapsto w_{1}-i\left(\sum_{j, k=1}^{m} \frac{\partial^{2} h_{1}}{\partial z_{j} \partial z_{k}}(0) z_{j} z_{k}+\sum_{j=1}^{d} \frac{\partial^{2} h_{1}}{\partial z_{1} \partial u_{j}}(0) z_{1} w_{j}+\sum_{j, k=1}^{d} \frac{\partial^{2} h_{1}}{\partial u_{j} \partial u_{k}}(0) w_{j} w_{k}\right)$,

we have

$$
h_{1}(z, u)=\sum_{j, k=1}^{m} a_{j, k} z_{j} \bar{z}_{k}+O_{3}(z, u)
$$

with $a_{1,1}=1$. In particular, we still have $\mathcal{L}_{M, 0}\left(\frac{\partial}{\partial x_{1}}\right)=\frac{\partial}{\partial u_{1}}$.

For a small $\varepsilon_{1}>0$, to be specified later, and a parameter $0 \leq t<\sqrt{\varepsilon_{1}}$, we let $U_{t}$ be the solutions of the parameter-dependent Bishop equation

$$
U_{t}=-\mathcal{T}_{0}\left(h\left(W_{t}, U_{t}\right)\right) \text {, where } W_{t}=(t \zeta, 0 \ldots, 0) .
$$

Since the data are smooth, we can solve this in the Hölder spaces $\mathcal{C}^{k, \alpha}\left(S^{1}, \mathbb{R}^{d}\right)(k \geq 1,0<\alpha<1)$ for $\varepsilon_{1}$ sufficiently small. Let the $A_{t}(\zeta)$ be the corresponding holomorphic disc and set $\gamma_{0}(s)=A_{\sqrt{s}}(0)$. One reads off from 
(3) that the curve $\gamma_{0}$ starts at the origin and runs in the space $i \mathbb{R}_{v}^{d}=\left\{z=u_{1}=\ldots=u_{n}=0\right\}$. It is shown in [HT] that (i) $\frac{d \gamma_{0}}{d s}(0)$ is a positive multiple of $\frac{\partial}{\partial v_{1}}$ and that (ii) $\gamma_{0}$ is on $\left[0, \varepsilon_{1}\right)$ as smooth as we please if $\varepsilon_{1}$ is sufficiently small.

The curves $\gamma_{p}$ will be obtained by varying the initial point in this construction. To this end we first produce a family of coordinates $\left(z_{p}, w_{p}\right)$ centered at $p$ such that $T_{p=0} M=\left\{v_{p}=0\right\}$. Clearly this can be achieved by an affine linear change of coordinates $\Phi_{p}$ which depends smoothly on $p$, where $p$ ranges in some $M$-neighborhood of the origin. Thus $\left(z_{p}, w_{p}\right)$ are holomorphic for $p$ fixed, but are only smooth in $p$. Next we rotate and dilate in such a way that $d \Phi_{p}(X)$ transforms to $\frac{\partial}{\partial x_{1}}$ at the origin and such that $\mathcal{L}_{M, 0}\left(\frac{\partial}{\partial x_{1}}\right)=\frac{\partial}{\partial u_{1}}$. Then we modify $\left(z_{p}, w_{p}\right)$ again as before in order to simplify second-order terms. Clearly all this can be done by a family of local biholomorphisms depending smoothly on the parameter $p$. In these last coordinates, which we still denote by $\left(z_{p}, w_{p}\right)$, we construct a curve $\tilde{\gamma}_{p}(s)$, $0 \leq s<\varepsilon_{1}$, verbatim by the same construction as above. Thus $\tilde{\gamma}_{p}(s)$ starts at the origin in $\left(z_{p}, w_{p}\right)$-space, which corresponds to the point $p$, and its time derivative at $s=0$ is a positive multiple of $\frac{\partial}{\partial v_{1}}$. The desired curve $\gamma_{p}$ with $\gamma_{p}(0)=p$ is then obtained by reversing the coordinate transformations. The nature of the process implies that $\frac{d \gamma_{p}}{d s}(0)$ is a positive multiple of $X(p)$.

We claim that $\tilde{M}^{+} \cup W=\bigcup_{p \in W, 0 \leq s<\varepsilon_{1}} \gamma_{p}(s)$ is a manifold with boundary $W$ attached to $M$ along $W$, provided $W$ is a sufficiently small open neighborhood of the origin in $M$, and $\varepsilon_{1}$ is sufficiently small. Actually, optimal regularity results for the Bishop equation yield that the solution, which depends on a finite dimensional set of parameters, has arbitrarily small loss of smoothness with respect to the parameters ([Tu2], see also [MP4]). Thus we have that data depending $\mathcal{C}^{k, \alpha}$-smoothly on all variables and parameters lead to $\mathcal{C}^{k, \alpha-0}$-smooth solutions. As the smoothness in $s$ is as good as needed and we can assume $k$ as large as we please, the mapping $(p, s) \mapsto \gamma_{p}(s)$ is as smooth as we please. First, the inverse function theorem implies the claim about $\tilde{M}^{+} \cup W$. Second, we may extend $(p, s) \mapsto \gamma_{p}(s)$ to $W \times\left(-\eta, \varepsilon_{1}\right)$ for some $0<\eta \ll 1$, and obtain an extended manifold $\tilde{M}=\bigcup_{p \in W,-\eta<s<\varepsilon_{1}} \gamma_{p}(s)$ as required in (a). The before mentioned fact that

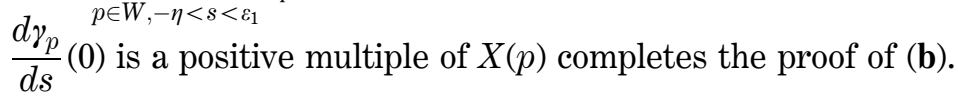


The proof of (c) is standard. One chooses an $M$-neighborhood $W^{\prime} \subset \subset U$ of $p_{0}$ so small that every $u \in C R(U)$ can be uniformly approximated on $\overline{W^{\prime}}$ by holomorphic polynomials $P_{j}$, using the Baouendi-Treves approximation theorem [BT1]. Restricting domains again, one restricts the above construction using only dises attached to $W^{\prime}$. Then the maximum modulus principle applied to the discs implies that the $P_{j}$ converge uniformly on $\tilde{M}^{+} \cup W$ to a continuous function $u_{+}$, which is $\mathrm{CR}$ on $\tilde{M}^{+}$. The proof of Proposition 3.2 is complete

Proof of Proposition 3.3. - Let us first sketch the geometrical idea. Since $Y=\mathcal{L}_{\tilde{M}, p_{0}}(X)$ is not complex tangent to $\tilde{M}$, it is classical (see [HT]) that appropriate $\mathcal{C}^{2, \alpha}$-small discs attached near $p_{0}$ to $\tilde{M}$ whose $z$-coordinates are parallel to $\mathrm{C} X$ are nontangent to $\tilde{M}$ along their boundaries, sticking out along directions which are approximately $Y$ modulo $H_{p_{0}} \tilde{M}$. This would be enough in order to extend CR functions defined on all of $\tilde{M}$. In the case at hand, we are only allowed to use discs attached to $\tilde{M}^{+} \cup W$. We will construct a family of dises whose boundaries touch $M$ quadratically in exactly one point and obtain CR extension to a $(\operatorname{dim} M+1)$-dimensional manifold (distinct from $\tilde{M}^{+}$) contained in the union of the discs. Since essentially the same construction is explained in great detail in [MP3, Section 5], it will suffice to give a concise review of what has to be done.

We will first construct a single disc attached to $\tilde{M}^{+} \cup W$ whose boundary touches $M$ at $p_{0}$. Choose local coordinates

$$
z_{1}, \ldots, z_{m+1}, w_{1}=u_{1}+i v_{1}, \ldots, w_{d-1}=u_{d-1}+i v_{d-1}
$$

centered at $p_{0}$ such that $\tilde{M}$ is locally given as a graph $v=\tilde{h}(z, u)$, with $\tilde{h}(0)=0, d \tilde{h}(0)=0$. After convenient rotations, we may assume that $X=\frac{\partial}{\partial x_{1}}, \mathcal{L}_{\tilde{M}, 0} X=\frac{\partial}{\partial u_{1}} \bmod H_{0} \tilde{M}$ and $T_{0} M=i \mathbb{R}_{y_{1}} \oplus \mathbb{C}_{z_{2}, \ldots, z_{m+1}}^{m} \oplus \mathbb{R}_{u_{1}, \ldots, u_{d-1}}^{d-1}$ (by multiplying by some $\zeta \in S^{1}$, we have rotated $X$ so that $\left.J X \in T_{0} M\right)$ ). For technical reasons, we also arrange that

$$
\tilde{h}\left(z_{1}, 0, \ldots, 0, u_{1} \ldots, u_{d-1}\right)=c\left|z_{1}\right|^{2}+O\left(|(z, u)|^{3}\right), \quad c>0,
$$

by eliminating pure terms of second order.

For $Z_{r}(\zeta)=(r(1-\zeta), 0, \ldots, 0), 0<r \ll 1$, let

$$
A_{r}(\zeta)=\left(Z_{r}(\zeta), U_{r}(\zeta)+i V_{r}(\zeta)\right)=\left(Z_{r}(\zeta), U_{r}(\zeta)+i h\left(Z_{r}(\zeta), U_{r}(\zeta)\right)\right)
$$

be the analytic disc obtained by solving the Bishop equation

$$
U_{r}=-\mathcal{T}_{1}\left(h\left(Z_{r}, U_{r}\right)\right) \text {. }
$$


Note that $A_{r}(1)=0$. The crucial point is that $r(1-\zeta)$ lies in the right $z_{1^{-}}$ halfplane and touches the imaginary axis quadratically at the origin, and that the curvature of its boundary at the origin becomes large for $r \rightarrow 0$. First, it is proved in [MP3, 5.5] that for $r>0$ small, we have $A_{r}(\bar{\triangle} \backslash\{1\}) \subset \tilde{M}^{+}$and that $A_{r}\left(S^{1}\right)$ touches $M$ quadratically at 0 . There is a geometric estimate for admissible $r$ which is stable under $\mathcal{C}^{2, \alpha}$-small deformations of $\tilde{h}$. Second, the usual classical argument (see [HT]) based on (4) shows that

$$
-\left.\frac{\partial V_{r}}{\partial \lambda}\right|_{\zeta=1}=r\left(c^{\prime}, 0, \ldots, 0\right)+o(r),
$$

with some $c^{\prime}>0$ independent of $r$ (here $\zeta=\lambda+i \eta$ ).

Let us take for granted, for the moment, that we can construct for a small fixed $r$ a $(\operatorname{dim} M+1)$-dimensional manifold $\hat{M}^{+}$attached to $M$, and containing the image $A_{r}((1-\varepsilon, 1))$ of the segment $(1-\varepsilon, 1)$, such that CR functions extend from $M$ to $\hat{M}^{+}$. Then (6) yields for the normalized outgoing direction at the origin

$$
-\frac{\partial v_{r}}{\partial \lambda}(1) /\left|\frac{\partial v_{r}}{\partial \lambda}(1)\right| \rightarrow\left(c^{\prime}, 0, \ldots, 0\right), \text { as } r \rightarrow 0 .
$$

But this implies that we can approximate the element defined by $\frac{\partial}{\partial v_{1}}$ in $T_{0} M / H_{0} M$ by directions of CR extensions. Since $\tilde{\xi}\left(\frac{\partial}{\partial v_{1}}\right)>0$ we have found a direction of extension $Z$ as desired. Now since a sufficiently small $r>0$ has been fixed, we drop it from the notation and write $A$.

It remains (i) to construct the manifold $\hat{M}^{+}$and (ii) to establish CR extension from $M$ to $\hat{M}^{+}$. The method for getting (i) is very similar to that of the proof of Proposition 3.2. We construct a family of local holomorphic coordinates

$$
z_{1, p}, \ldots, z_{m+1, p}, w_{1, p}, \ldots, w_{d-1, p}
$$

coinciding with the above coordinates for $p=0$ and satisfying the following properties:

a) $\left(z_{p}, w_{p}\right)$ depend $\mathcal{C}^{3}$-smoothly on the parameter $p$, which ranges in a small $M$-neighborhood $W^{\prime \prime}$ of the origin.

b) For $p$ fixed, $\left(z_{p}, w_{p}\right)$ are holomorphic and centered at $p$, and we have $T_{0} M=i \mathbb{R}_{y_{1, p}} \oplus \mathbb{C}_{z_{2, p}, \ldots, z_{m+1, p}}^{m} \oplus \mathbb{R}_{u_{1, p}, \ldots, u_{d-1, p}}^{d-1}$.

We apply the above construction with dependence on the parameter $p$. This yields dises $A_{p}$ attached to $\tilde{M}^{+} \cup W^{\prime}$ and touching $M$ quadratically at $A_{p}(1)=p$. Using regularity results for the Bishop equation ([Tu2], also 
[MP4]), we see that $A_{p}(\zeta)$ depends $\mathcal{C}^{2,1 / 2}$-smoothly on $p$ and $\zeta \in \bar{\Delta}$. After a further shrinking of $W^{\prime \prime}$ and with a smaller $\varepsilon$, we obtain a manifold $\hat{M}^{+} \cup W^{\prime \prime}=\bigcup_{p \in W^{\prime \prime}} A_{p}((1-\varepsilon, 1])$ as required in (i).

To show (ii) we just have to remember from the construction of $\tilde{M}^{+}$that every $u \in C R(U)$ can be approximated by holomorphic polynomials $P_{j}$ which converge uniformly on $\overline{W^{\prime}} \cup \tilde{M}^{+}$. Hence the $P_{j}$ also converge on $\hat{M}^{+} \cup W^{\prime \prime}$ to a continuous CR function extending $u$. This yields (ii) and completes the proofs of Proposition 3.3, and also of Theorems 1.1 and 2.2

\section{Applications to CR meromorphic functions.}

One of our motivations is to find concrete applications for the Siegeltype theorems proved in [HN2], [HN3]. In these papers, far reaching global consequences for the field of CR meromorphic functions are proved for CR manifolds satisfying a local extension property E. For a $\mathcal{C}^{\infty}$-smooth generic CR submanifold $M$ of a complex manifold $N$, property E means that the canonical restriction mapping $\mathcal{O}_{N, p} \rightarrow \mathcal{C R}_{M, p}$ is surjective for every $p \in M$. Here $\mathcal{O}_{N}$ and $\mathcal{C R}_{M}$ denote the sheaf of germs of holomorphic functions on $N$ and the sheaf of germs of $\mathcal{C}^{\infty}$-smooth CR functions on M, respectively, and $\mathcal{O}_{N, p}, \mathcal{C} \mathcal{R}_{M, p}$ are their stalks at $p$. We obtain immediately that a manifold satisfying the assumptions of Theorem 1.1 in a coordinate neighborhood of each of its points has property E. Hence we obtain all results proved in [HN2], [HN3] by carrying the local situation studied here to general manifolds.

Actually the main results in [HN2], [HN3] concern CR meromorphic functions rather than CR functions. Similarly as ordinary meromorphic functions, we define $C R$ meromorphic functions in the usual sense on $U \subset M$ as functions which are defined on a dense open subset of $U$ and can be represented near every point $p \in U$ as the quotient $p / q$ of $\mathcal{C}^{\infty}$-smooth CR functions $p, q$, where $q$ does not vanish identically on any nonempty open subset. If $M$ has property E, every CR meromorphic function on $U$ is the restriction of a meromorphic function defined on some ambient neighborhood of $U$ in $N$.

Let $M$ be a smooth compact locally embeddable CR manifold of CR dimension $m$ and CR codimension $d$, which at each point satisfies the hypotheses of Theorem 1.1. Then the field $\mathcal{K}(M)$ of CR meromorphic functions on $M$ has transcendence degree $k \leq m+d$. If $f_{1}, \ldots, f_{k}$ is a maximal set of algebraically independent $\mathrm{CR}$ meromorphic functions on $M$, then 
$\mathcal{K}(M)$ is a simple finite algebraic extension of the field $\mathbb{C}\left(f_{1}, \ldots, f_{k}\right)$ of rational functions of the $f_{1}, f_{2}, \ldots, f_{k}$. Assuming that $M$ is connected, there is also an equivalence between the algebraic dependence over $\mathbb{C}$, and the analytic dependence, of a finite set of CR meromorphic functions in $\mathcal{K}(M)$. When $M$ has a projective embedding there is an analogue of Chow's theorem, and $\mathcal{K}(M)$ is isomorphic to the field $\mathcal{R}(Y)$ of rational functions on an irreducible projective algebraic variety $Y$, and $M$ has a CR embedding in reg $Y$. For details, and further applications and remarks, see [HN2], [HN3].

\section{CR meromorphic mappings according to Harvey and Lawson}

An alternative notion of $\mathrm{CR}$ meromorphic functions and mappings was suggested by Harvey and Lawson in the context of the complex Plateau problem and studied in [HL], [DH], [DS], [MP1], [MP2]. The following definitions appear in [HL] for hypersurfaces and in [DS] for CR manifolds of arbitrary codimension. Let $M$ be a smooth generic CR submanifold of a complex manifold $N$ of $\mathrm{CR}$ dimension $m$ and codimension $d$, and let $X$ be an arbitrary complex manifold. Then a $C R$ meromorphic mapping $F$ in the sense of Harvey-Lawson of an open $U \subset M$ with values in $X$ is given by a triple $\left(F, \mathcal{D}_{F}, \Gamma_{F}\right)$ with the following properties:

(a) $\mathcal{D}_{F}$ is an open dense subset of $U$,

(b) $F: \mathcal{D}_{F} \rightarrow X$ is a $\mathcal{C}^{1}$-smooth CR mapping,

(c) the closure of the graph of $F$ in $U \times X$ equals $\Gamma_{F}$ and is a local scarred CR cycle of $\mathrm{CR}$ dimension $m$ and dimension $2 m+d$ in $N \times X$.

In (c) we mean that $\Gamma_{F}$ is of locally finite $(\operatorname{dim} M)$-dimensional Hausdorff measure and contains a closed subset $\sigma$ (the scar set) of $(\operatorname{dim} M)$ dimensional Hausdorff measure zero such that (i) $\Gamma_{F} \backslash \sigma$ is a $\mathcal{C}^{1}$-smooth CR manifold of same dimension and CR dimension as $M$ and (ii) in a neighborhood of every $(p, x) \in \Gamma_{F}$, integration over $\Gamma_{F} \backslash \sigma$ yields a closed current (see [HL], [DS], [MP1] for full details). If $X$ equals $\mathrm{P}^{1}$, the complex projective line, we also speak of CR meromorphic functions in the sense of Harvey-Lawson.

Meromorphic extension of these CR meromorphic mappings is technically complicated because of the presence of the scar set. Actually it requires a certain machinery to derive the counterpart of Theorem 1.1 for 
CR meromorphic functions in the sense of Harvey-Lawson. We can prove this for all manifolds with property E.

THEOREM 6.1. Let $M$ be a smooth generic CR submanifold of a complex manifold $N$ with property $E$. Then every $C R$ meromorphic function $F$ in the sense of Harvey-Lawson, defined on an open set $U \subset M$ and with values in $\mathbb{P}^{1}$ has a meromorphic extension $\tilde{F}$ to a neighborhood $V$ of $U$ in $N$. More precisely, the graph $\Gamma_{\tilde{F}} \subset V \times \mathbb{P}^{1}$ of $\tilde{F}$ satisfies $\Gamma_{\tilde{F}} \cap\left(U \times \mathbb{P}^{1}\right)=\Gamma_{F}$. In particular, $F$ is a CR meromorphic function in the usual sense.

A CR manifold $M$ is called minimal at a point $p \in M$ (in the sense of Tumanov) if there is no germ of a CR manifold $N \subset M$ of the same CR dimension as $M$ and of lower dimension than $M$ containing $p$.

Lemma 6.2. Let $M$ be a smooth generic $C R$ manifold in $\mathrm{C}^{n}$ with property $E$. Then $M$ is minimal at every point $p \in M$.

Proof. Let us assume that $M$ is not minimal at $p_{0}$. By $[\mathrm{BR}]$ there is a smooth CR function $u$ defined on an open neighborhood $U^{\prime} \subset U$ of $p_{0}$ which does not extend holomorphically to any open wedge attached to $M$ near $p_{0}$, in contradiction to property $\mathrm{E}$.

Proof of Theorem 6.1. - Let $F$ be a $\mathbb{P}^{1}$-valued CR meromorphic function in the sense of Harvey-Lawson defined on $U \subset M$. First we construct a local extension of $F$ to an ambient neighborhood of a given $p_{0} \in U$. Because of Lemma 6.2, [MP2, Theorem 1.2] gives meromorphic extension to an open wedge $\mathcal{W}$ attached to a neighborhood $U^{\prime}$ of $p_{0}$ in $M$. More precisely, there is an open truncated cone $C \subset \mathbb{C}^{n}$ with vertex at the origin and a meromorphic function $\tilde{F}$ on $\mathcal{W}=U^{\prime}+C$ which attains $F$ as continuous boundary value on $\mathcal{D}_{F}$.

From property E and a Baire category argument (see [HN2]), it follows that smooth CR functions on $U^{\prime}$ extend holomorphically to a uniform ambient neighborhood $V^{\prime}$ of $U^{\prime}$. Since the envelope of meromorphy coincides with the envelope of holomorphy for domains in $\mathrm{C}^{n}$, functions which are meromorphic on an arbitrarily thin neighborhood of $U^{\prime}$ extend meromorphically to $V^{\prime}$. For fixed $c \in C$, the rigid translates $U_{\varepsilon}^{\prime}=U^{\prime}+\{\varepsilon c\}$ approach $U^{\prime}$ for $\varepsilon \downarrow 0$. To obtain the desired extension to a neighborhood of $p_{0}$, it suffices to choose $\varepsilon \in(0,1)$ so small that $p_{0} \in V^{\prime}+\{\varepsilon c\}$ and to extend $\tilde{F}$ to $V^{\prime}+\{\varepsilon c\}$. 
Now a standard gluing argument yields a meromorphic function $\tilde{F}$ which is defined on a neighborhood $V$ of $U$ in $\mathbb{C}^{n}$ and coincides with $F$ on $\mathcal{D}_{F}$. It remains to prove that $\Gamma_{\tilde{F}} \cap\left(U \times \mathbb{P}^{1}\right)=\Gamma_{F}$. Near points in $\mathcal{D}_{F}$ this is obvious. From (b) in the definition of CR meromorphic mappings and the corresponding (well known) property of meromorphic functions, we deduce $\Gamma_{F} \subset \Gamma_{\tilde{F}} \cap\left(U \times \mathbb{P}^{1}\right)$. Assume that there is $\left(p_{0}, \zeta_{0}\right) \in \Gamma_{\tilde{F}} \cap\left(U \times \mathbb{P}^{1}\right) \backslash \Gamma_{F}$. This is obviously impossible if $p_{0}$ is a point near which $\tilde{F}$ is a smooth mapping. Hence it remains to consider the case in which $p_{0}$ lies in the indeterminacy set $\Sigma_{\tilde{F}}=\left\{p:\{p\} \times \mathbb{P}^{1} \subset \Gamma_{\tilde{F}}\right\}$.

Following [DS], we also consider the indeterminacy set $\Sigma_{F}$ of $F$ defined by

$$
\Sigma_{F}=\left\{p \in U:\{p\} \times \mathbb{P}^{1} \subset \Gamma_{F}\right\} .
$$

Our assumption on $p_{0}$ means that $p_{0} \notin \Sigma_{F}$. Let $\alpha$ be a biholomorphism of $\mathbb{P}^{1}$ mapping $\zeta_{0}$ to $\infty$. It is observed in [DS] that the set-valued function $\alpha \circ F$ naturally induces a CR distribution $g$ of order one on a neighborhood $U_{p_{0}}$ of $p_{0}$ in $M$. As $M$ is minimal in $p_{0}$, Tumanov's theorem [Tu1] and the usual extension techniques for CR distributions yield holomorphic extension to an open wedge attached to $M$ at $p_{0}$ (assuming $g$ as boundary value in the weak sense). Then an argument with approach manifolds as above yields a holomorphic extension $\tilde{g}$ to a full neighborhood of $p_{0}$. Observing that $\alpha \circ F$ and $\tilde{g}$ are smooth and coincide at points of $\mathcal{D}_{F}$, we obtain that the equality $\tilde{F}=\alpha^{-1} \circ \tilde{g}$ holds near $p_{0}$. In particular, $\tilde{F}$ is smooth near $p_{0}$, in contradiction to $p_{0} \in \Sigma_{\tilde{F}}$. The proof of Theorem 6.1 is complete

REMARK 6.3. It requires only little extra work to derive a corresponding result for CR meromorphic mappings with values in a projective manifold $X$. Note that in the general case we can only expect $\Gamma_{F} \subset \Gamma_{\tilde{F}} \cap(U \times X)$.

\section{Homogeneous examples}

At first glance, it may seem hard to find examples of CR manifolds of kind 3 satisfying the conditions of Theorem 1.1. However, the theory of homogeneous CR manifolds provides many of them in a very natural way (see [MN1], [MN2], [MN3], [MN4], [AMN]). We give a concise description of a class of such homogeneous CR manifolds, and refer to [AMN] for more details.

Let $G^{\mathrm{C}}$ be a complex connected semisimple Lie group, with Lie algebra $\mathrm{g}^{\mathrm{C}}$, and $G$ a connected real form of $G^{\mathrm{C}}$, with Lie algebra $\mathrm{g}$. Fix a Cartan 
subgroup $H$ of $G$, which is maximally noncompact, that is a Cartan subgroup such that a maximal compact torus in $H$ has minimal dimension, and denote by $\mathfrak{h}$ and $\mathfrak{h}^{\mathrm{C}}$ the Lie algebras of $H$ and of its complexification $H^{\mathrm{C}}$. In the set of roots $\mathcal{R}=\mathcal{R}\left(\mathfrak{g}^{\mathrm{C}}, \mathfrak{h}^{\mathrm{C}}\right)$ choose a subset $\mathcal{R}^{+}$of positive roots, adapted to $\mathfrak{g}$ (cf. [AMN], Proposition 6.1), and let $\mathcal{B}$ be the corresponding set of positive simple roots. To any subset $\Phi \subset \mathcal{B}$ we associate the parabolic subalgebra and subgroup

$$
\begin{aligned}
\mathfrak{q}_{\Phi} & =\mathfrak{h}^{\mathrm{C}}+\sum_{\alpha \in \mathcal{R}^{+}} \mathfrak{g}_{\alpha}^{\mathrm{C}}+\sum_{\substack{\alpha \in \mathcal{R}^{+} \\
(\operatorname{supp} \alpha) \cap \phi=\emptyset}} \mathfrak{g}_{\alpha}^{\mathrm{C}}, \\
Q_{\Phi} & =\operatorname{Norm}_{G^{\mathrm{C}}}\left(\mathfrak{q}_{\Phi}\right) .
\end{aligned}
$$

Here $\mathrm{g}_{\alpha}^{\mathrm{C}}$ is the eigenspace in $\mathrm{g}^{\mathrm{C}}$ of a root $\alpha$, and supp $\alpha$ is the support of a root $\alpha$ in $\mathcal{B}$. The group $Q_{\Phi}$ has Lie algebra $\mathfrak{q}_{\Phi}$.

The group $G^{\mathrm{C}}$ acts via the adjoint representation on $\mathrm{g}^{\mathrm{C}}$ and on linear subspaces of $\mathfrak{g}^{\mathrm{C}}$. Fix a subset $\Phi \subset \mathcal{B}$ and let $d$ be the dimension of $\mathfrak{a}_{\Phi}$. The orbit

$$
Y=\left\{\operatorname{Ad}_{\mathfrak{g}^{\mathrm{C}}}(g)\left(\mathfrak{q}_{\Phi}\right) \mid g \in G^{\mathrm{C}}\right\} \subset \operatorname{Gr}_{d}\left(\mathfrak{g}^{\mathrm{C}}\right)
$$

through $\mathfrak{q}_{\Phi}$ in the Grassmannian of $d$-planes in $\mathrm{g}^{\mathrm{C}}$ is the flag manifold of parabolic subalgebras of $\mathrm{g}^{\mathrm{C}}$ conjugate by an inner automorphism to $\mathfrak{q}_{\Phi}$. It is a smooth irreducible projective subvariety of $\operatorname{Gr}_{d}\left(\mathrm{~g}^{\mathrm{C}}\right)$, isomorphic to $G^{\mathrm{C}} / Q_{\Phi}$. The orbit

$$
M=\left\{\operatorname{Ad}_{\mathfrak{g}^{\mathrm{c}}}(g)\left(\mathfrak{q}_{\Phi}\right) \mid g \in G\right\} \subset \operatorname{Gr}_{d}\left(\mathfrak{g}^{\mathrm{C}}\right)
$$

of $G$ through $\mathfrak{q}_{\Phi}$ is a smooth generic CR submanifold of $Y$ which, by our choice of the Cartan subgroup and of the system of positive roots, is compact, and is called the minimal orbit of $G$ in $Y$.

If all local CR functions near a point $p$ of $M$ extend to a full neighborhood of $p$ in $Y$, then the pair $(M, Y)$ has property $\mathrm{E}$ of [HN2], hence the field $\mathcal{K}(M)$ of $C R$ meromorphic functions is isomorphic to the field $\mathcal{R}(Y)$ of rational functions on $Y$, because $Y$ is the smallest projective variety containing $M$ (see [HN2]).

Example 7.1. Identify $C^{6}$, with the standard basis $\left\{\mathrm{e}_{j}\right\}_{1 \leq j \leq 6}$, with the quaternionic vector space $\mathbb{H}^{3}$ by setting, for $\lambda \in \mathrm{C}$,

$$
\boldsymbol{j} \lambda \mathrm{e}_{2 j-1}=\bar{\lambda} \mathrm{e}_{2 j}, \quad \boldsymbol{j} \lambda \mathrm{e}_{2 j}=-\bar{\lambda} \mathrm{e}_{2 j-1} .
$$

Consider the complex flag manifold

$$
Y=\left\{\ell_{1} \subset \ell_{3} \subset \ell_{5} \subset \mathbb{C}^{6} \mid \operatorname{dim} \ell_{2 j-1}=2 j-1,1 \leq j \leq 3\right\} .
$$


Then $Y$ is a compact 13-dimensional complex manifold, homogeneous for the action of $G^{\mathrm{C}}=\mathrm{SL}(6, \mathrm{C})$. Near the point

$$
o=\left(\left\langle\mathrm{e}_{1}\right\rangle_{\mathrm{C}},\left\langle\mathrm{e}_{1}, \mathrm{e}_{2}, \mathrm{e}_{3}\right\rangle_{\mathrm{C}},\left\langle\mathrm{e}_{1}, \mathrm{e}_{2}, \mathrm{e}_{3}, \mathrm{e}_{4}, \mathrm{e}_{5}\right\rangle_{\mathrm{C}}\right)
$$

it admits a holomorphic chart given by the nonconstant entries of the matrix

$$
A=\left(\begin{array}{ccccc}
1 & 0 & 0 & 0 & 0 \\
z_{1} & 1 & 0 & 0 & 0 \\
z_{2} & 0 & 1 & 0 & 0 \\
z_{3} & z_{6} & z_{9} & 1 & 0 \\
z_{4} & z_{7} & z_{10} & 0 & 1 \\
z_{5} & z_{8} & z_{11} & z_{12} & z_{13}
\end{array}\right) .
$$

Let $M$ be the real submanifold of $Y$ given by

$$
\left.M=\left\{\left(\ell_{1}, \ell_{3}, \ell_{5}\right) \in Y \mid \mathbb{H} \ell_{1} \subset \ell_{3}, \mathbb{H} \ell_{3} \subset \ell_{5}\right)\right\} .
$$

Then $M$ is homogeneous for the action of the real form $G=\mathrm{SL}(3, \mathbb{H})$ of $G$, and it is a compact real-analytic homogeneous generic CR submanifold of $Y$. Denoting by $A_{j}$ the $j$-th column of $A$, near the point $o$ the manifold $M$ is defined by the system of equations

$$
\left\{\begin{array}{l}
\operatorname{rk}\left(A_{1}, A_{2}, A_{3}, \mathbf{j} A_{1}\right)=3, \\
\operatorname{rk}\left(A_{1}, A_{2}, A_{3}, A_{4}, A_{5}, \mathbf{j} A_{1}, \mathbf{j} A_{2}, \mathbf{j} A_{3}\right)=5,
\end{array}\right.
$$

which, in the coordinates $\left\{z_{j}\right\}_{1 \leq j \leq 13}$, are

$$
\left\{\begin{array}{l}
z_{2}-\bar{z}_{6}+z_{1} \bar{z}_{3}+z_{3} \bar{z}_{9}-z_{1} \bar{z}_{1} \bar{z}_{6}-z_{1} \bar{z}_{2} \bar{z}_{9}=0, \\
z_{4}-\bar{z}_{8}+z_{1} \bar{z}_{5}+z_{3} \bar{z}_{11}-z_{1} \bar{z}_{1} \bar{z}_{8}-z_{1} \bar{z}_{2} \bar{z}_{10}=0, \\
z_{10}-\bar{z}_{12}+z_{9} \bar{z}_{11}+z_{11} \bar{z}_{13}-z_{9} \bar{z}_{9} \bar{z}_{12}-z_{9} \bar{z}_{10} \bar{z}_{13}=0, \\
z_{5}+\bar{z}_{7}-z_{1} \bar{z}_{4}-z_{3} \bar{z}_{10}+z_{1} \bar{z}_{1} \bar{z}_{7}+z_{1} \bar{z}_{2} \bar{z}_{10}=0,
\end{array}\right.
$$

thus $M$ has CR dimension 5 and codimension 8. The holomorphic tangent space $T_{o}^{1,0} M$ admits the basis $\left\{\partial / \partial z_{j}\right\}_{j=1,3,9,11,13}$. The space of Levi forms has real dimension 6 , and in the basis $\left\{\partial / \partial z_{j}\right\}_{j=1,3,9,11,13}$ it is the space of Hermitian symmetric matrices of the form

$$
\left(\begin{array}{lllll}
0 & \alpha & 0 & 0 & 0 \\
\bar{\alpha} & 0 & \alpha & \beta & 0 \\
0 & \bar{\alpha} & 0 & \gamma & 0 \\
0 & \bar{\beta} & \bar{\gamma} & 0 & \gamma \\
0 & 0 & 0 & \bar{\gamma} & 0
\end{array}\right), \quad \alpha, \beta, \gamma \in \mathbb{C}
$$

as the fourth equation does not contribute to the Levi form. In some 
characteristic codirections the Levi forms are zero, but in the remaining characteristic codirections, the Levi forms all have signature $(1,1)$ or signature $(2,2)$, and $M$ is trace pseudoconcave. Moreover $M$ has kind 3, indeed $G_{2}$ has real dimension 16 and $G_{3}$ is the whole tangent space of $M$. By Theorem 1.1 germs of $\mathrm{CR}$ distributions on $M$ are real-analytic and extend to germs of holomorphic functions on $Y$.

With the notation of [AMN], $M$ is the minimal orbit of the simple Lie group of type $\mathrm{AII}_{5}$ associated to the parabolic subalgebra $\mathfrak{q}=\mathfrak{q}_{\left\{\alpha_{1}, \alpha_{3}, \alpha_{5}\right\}}$.

ExAmple 7.2. The example above can be generalized to the following pairs of complex flag manifolds, homogeneous for the action of SL $(2 n, \mathrm{C})$, and compact generic CR submanifolds, homogeneous for the action of $\mathrm{SL}(n, \mathrm{H})$ :

$$
\left\{\begin{array}{l}
Y=\left\{\ell_{2 j_{1}-1} \subset \ell_{2 j_{2}-1} \subset \ell_{2 j_{3}-1} \subset \mathbb{C}^{2 n} \mid \operatorname{dim} \ell_{2 j_{k}-1}=2 j_{k}-1,1 \leq k \leq 3\right\} \\
M=\left\{\left(\ell_{2 j_{1}-1}, \ell_{2 j_{2}-1}, \ell_{2 j_{3}-1}\right) \in Y \mid \begin{array}{ll}
\operatorname{dim}\left(\ell_{2 j_{k}-1} \cap \mathrm{H} \ell_{2 j_{k}-1}\right)=2 j_{k}-2, & 1 \leq k \leq 3 \\
\mathrm{H} \ell_{2 j_{k}-1} \subset \ell_{2 j_{k+1}-1}, & 1 \leq k \leq 2
\end{array}\right. \\
\text { for } 1=j_{1}<j_{2}<j_{3} \leq n .
\end{array},\right.
$$

In this case the complex dimension of $Y$, the $\mathrm{CR}$ dimension of $M$, and the $\mathrm{CR}$ codimension of $M$ are:

$$
\begin{aligned}
\operatorname{dim}_{\mathrm{C}} Y & =(2 n-1)+2\left(j_{2}-1\right)\left(2 n-2 j_{2}+1\right)+2\left(j_{3}-j_{2}\right)\left(2 n-2 j_{3}+1\right), \\
\operatorname{dim}_{\mathrm{CR}} M & =2 n+2 j_{3}-7, \\
\operatorname{codim}_{\mathrm{CR}} M & =4\left(\left(n-j_{3}\right)\left(j_{3}-1\right)+\left(j_{3}-j_{2}\right)\left(j_{2}-1\right)+1\right) .
\end{aligned}
$$

The space of Levi forms of $M$ has dimension

$$
\begin{aligned}
\left(2 j_{2}-3\right)\left(2 j_{3}-2 j_{2}+1\right)+\left(2 j_{3}-2 j_{2}-1\right)\left(2 n-2 j_{3}+\right. & \left.2 j_{2}-1\right)+ \\
& +\left(2 n-2 j_{3}\right)\left(2 j_{3}-2 j_{2}\right),
\end{aligned}
$$

and all of them, in a suitable basis (as described in [AMN]), have all diagonal entries equal to zero. Hence $M$ is trace pseudoconcave, and has not kind 2. It can be checked that $M$ has kind 3, thus $M$ satisfies the hypotheses of Theorem 1.1 and germs of CR distributions extend holomorphically to a full neighborhood.

With the notation of [AMN] $M$ is the minimal orbit of the simple Lie group of type $\mathrm{A} \mathrm{II}_{2 n-1}$ associated to the parabolic subalgebra $\mathfrak{q}=\mathfrak{q}_{\left\{\alpha_{1}, \alpha_{2 j_{2}-1}, \alpha_{2_{3}-1}\right\}}$. By duality, a completely analogous statement holds for the case $1 \leq j_{1}<j_{2}<j_{3}=n$. 
EXAMPLE 7.3. Similar to the previous one is the case of pairs of complex flag manifolds, homogeneous for the action of SL(2n, C), and compact generic $\mathrm{CR}$ submanifolds, homogeneous for the action of $\mathrm{SL}(n, \mathbb{H})$ :

$$
\left\{\begin{array}{l}
Y=\left\{\ell_{2 j_{1}-1} \subset \ell_{2 j_{2}-1} \subset \mathbb{C}^{2 n} \mid \operatorname{dim} \ell_{2 j_{k}-1}=2 j_{k}-1, k=1,2\right\}, \\
M=\left\{\left(\ell_{2 j_{1}-1}, \ell_{2 j_{2}-1}\right) \in Y \mid \begin{array}{l}
\operatorname{dim}\left(\ell_{2 j_{k}-1} \cap \mathbb{H} \ell_{2 j_{k}-1}\right)=2 j_{k}-2, \quad k=1,2 \\
\mathbb{H} \ell_{2 j_{1}-1} \subset \ell_{2 j_{2}-1}
\end{array}\right. \\
\text { for } \quad 1<j_{1}<j_{2}<n .
\end{array},\right.
$$

Also in this case, $M$ has kind 3 and is trace pseudoconcave.

ExAmple 7.4. Let $G^{\mathrm{C}}$ be the connected and simply connected simple complex Lie group of type $F_{4}$, and let $\mathrm{g}^{\mathrm{C}}$ its Lie algebra. Fix a Cartan subalgebra $\mathfrak{h}^{\mathrm{C}}$ of $\mathrm{g}^{\mathrm{C}}$ and a system of simple roots $\mathcal{B}=\left\{\alpha_{j}\right\}_{1 \leq j \leq 4}$ of the root system $\mathcal{R}=\mathcal{R}\left(\mathfrak{g}^{\mathrm{C}}, \mathfrak{h}^{\mathrm{C}}\right)$ (we use the root numbering scheme of Bourbaki, see [AMN], Appendix). Let $\left\{\omega_{j}\right\}_{1 \leq j \leq 4}$ be the set of fundamental weights dual to $\mathcal{B}$ and let $V=V_{\omega_{2}}$ be the fundamental representation of $G^{\mathrm{C}}$ with highest weight $\omega_{2}$. Let $Y$ be the $G^{\mathrm{C}}$-orbit, in $\mathbb{P}(V)$, of the highest weight root space. With the notation of [AMN], $Y$ is the complex flag manifold of $G^{\mathrm{C}}$ consisting of parabolic subalgebras of $\mathrm{g}^{\mathrm{C}}$ that are conjugate to $\mathfrak{q}_{\left\{\alpha_{2}\right\}}$.

Inside $Y$ consider the minimal orbit $M$ of the real form of $G^{\mathrm{C}}$ of type F II (also denoted by $F_{4(-20)}$ ). It is a CR manifold of CR dimension 9 and CR codimension 11. By [AMN], Theorem 9.1, it is of finite type, and by [AMN], Theorem 13.5, it is trace pseudoconcave. Direct computation shows that $G_{2}$ has codimension 4 and $G_{3}$ is the whole tangent space of $M$, thus $M$ has kind 3 . Hence also in this case we have holomorphic extension for germs of $\mathrm{CR}$ distributions.

Acknowledgments. The second and the fourth author would like to thank the Mathematisches Institut der Humboldt-Universität zu Berlin, and Professor Jürgen Leiterer in particular, for their kind hospitality. Furthermore we thank Anna Siano for information about the literature.

\section{REFERENCES}

[AMN] A. Altomani - C. Medori - M. NaCinovich, The CR structure of minimal orbits in complex flag manifolds, J. Lie Theory, 16 (2006), pp. 483-530.

[BR] M. S. BaOUendi - L. P. Rothschild, Cauchy Riemann functions on manifolds of higher codimension in complex space, Invent. Math., 101 (1990), pp. 45-56. 
[BT1] M. S. BAOUENDI - F. TREVEs, A property of the functions and distributions annihilated by a locally integrable system of complex vector fields, Ann. Math., 113 (1981), pp. 387-421.

[BT2] M. S. Baouend - F. Treves, About the holomorphic extension of $C R$ functions on real hypersurfaces in complex space, Duke Math. J., 51, no. 1 (1984), pp. 77-107.

[B] E. BISHop, Differentiable manifolds in complex Euclidean space, Duke Math. J., 32 (1965), pp. 1-21.

[Bo] A. BogGess, CR extendability near a point where the first Levi form vanishes, Duke Math. J., 48 (1981), pp. 665-684.

[BP] A. Boggess - J. PolkIng, Holomorphic extensions of CR functions, Duke Math. J., 49 (1982), pp. 757-784.

[DS] T. C. DINH - F. SARKIS, Wedge removability of metrically thin sets and application to the CR meromorphic extension, Math. Z., 238 (2001), pp. 639-653.

[DH] P. Dolbeault - G. Henkin, Chaînes holomorphes de bord donné dans un ouvert q-concave de CP ${ }^{n}$, Bull. Soc. Math. France, 125 (1997), pp. 383-445.

[FR] J. E. FORNAESS - C. REA, Local holomorphic extendability and nonextendability of CR-functions on smooth boundaries, Ann. Scuola Norm. Sup. Pisa Cl. Sci. (4), 12, no. 3 (1985), pp. 491-502.

[HL] R. HARVEY - B. LaWson, On boundaries of complex analytic varieties, Part II, Ann. Math., 106 (1977), pp. 213-238.

[HN1] C. D. Hill - M. NAcinovich, A weak pseudoconcavity condition for abstract almost CR manifolds, Invent. Math., 142 (2000), pp. 251-283.

[HN2] C. D. Hill - M. Nacinovich, Fields of CR meromorphic functions, Rend. Sem. Mat. Univ. Padova, 111 (2004), pp. 179-204.

[HN3] C. D. HiLl - M. NACINOvich, Elementary pseudoconcavity and fields of CR meromorphic functions, Rend. Sem. Mat. Univ. Padova, 113 (2005), pp. 99-115.

[HN4] C. D. Hill - M. Nacinovich, Conormal suspensions of differential complexes, J. Geom. Anal., 10, 3 (2000), pp. 496-537.

[HT] C. D. Hill - G. TAIANi, Families of analytic discs in $\mathrm{C}^{n}$ with boundaries on a prescribed CR submanifold, Ann. Sc. Norm. Pisa, 5 (1978), pp. 327-380.

[J] B. JöRICKE, Deformation of CR-manifolds, minimal points and $C R$ manifolds with the microlocal analytic extension property, J. Geom. Anal., 6 (1996), pp. 555-611.

[MN1] C. MEDORI - M. NACINOviCH, Levi-Tanaka algebras and homogeneous CR manifolds, Compositio Mathematica, 109 (1997), pp. 195-250.

[MN2] C. Medori - M. Nacinovich, Classification of semisimple Levi-Tanaka algebras, Ann. Mat. Pura Appl., CLXXIV (1998), pp. 285-349.

[MN3] C. Medori - M. Nacinovich, Complete nondegenerate locally standard CR manifolds, Math. Ann., 317 (2000), pp. 509-526.

[MN4] C. Medori - M. NACinovich, Algebras of infinitesimal CR automorphisms, J. Algebra, 287 (2005), pp. 234-274.

[M] J. MERKER, Global minimality of generic manifolds and holomorphic extendibility of CR functions, IMRN, 8 (1994), pp. 329-342.

[MP1] J. Merker - E. Porten, On the local meromorphic extension of $C R$ meromorphic mappings, Ann. Polon. Math., 70 (1998), pp. 163-193. 
[MP2] J. Merker - E. Porten, Metrically thin singularities of integrable CR functions, Internat. J. Math., 11 (2000), pp. 857-872.

[MP3] J. MERKER - E. Porten, Characteristic foliations on maximally real submanifolds of $\mathbb{C}^{n}$ and removable singularities for $C R$ functions, IMRP, Volume 2006 (2006), Article ID 72069, pp.1-131.

[MP4] J. Merker - E. Porten, Holomorphic Extension of CR Functions, Envelopes of Holomorphy, and Removable Singularities, IMRS, Volume 2006 (2006), Article ID 28925, pp.1-286.

[NV] M. Nacinovich - G. VALLI,Tangential Cauchy-Riemann complexes on distributions, Ann. Mat. Pura Appl., 146 (1987), pp. 123-160.

[S] J. SJÖSTRAND, The FBI transform for CR submanifolds of $\mathbb{C}^{n}$, Prépublications Mathématiques Orsay 1982.

[T1] J.-M. TRÉPREAU, Sur le prolongement holomorphe des fonctions CR définies sur une hypersurface reelle de classe $C^{2}$, Invent. Math., 83 (1986), pp. 583-592.

[T2] J.-M. TRÉPREAU, Sur la propagation des singularités dans les variétés CR, Bull. Soc. Math. Fr., 118 (1990), pp. 403-450.

[T] F. Treves, Hypoanalytic Structures: Local Theory, Princeton Univ. Press 1992.

[Tu1] A. E. Tumanov, Extension of $C R$-functions into a wedge from a manifold of finite type, Math. USSR Sb., 64 (1989), pp. 129-140.

[Tu2] A. E. Tumanov, On the propagation of extendability of CR functions, Complex Analysis and Geometry, Proc. of the Conference in Trento 1995. Lecture Notes in Pure and Appl. Math., 173 (Dekker 1996), pp. 479-498.

Manoscritto pervenuto in redazione il 17 marzo 2009. 